\title{
Study of Clinical Predictive Value and Immune Characterization of SLFNI I in Clear Cell Renal Cell Carcinoma
}

Yifu Liu

Zhicheng Zhang

Shengqiang $\mathrm{Fu}$

Siyuan Wang

Xiaofeng Cheng

Kunyang Lei

Zhilong Li

Ting Sun

Ming Ma

Department of Urology, The First Affiliated Hospital of Nanchang University, Nanchang, 330006, Jiangxi

Province, People's Republic of China
Correspondence: Ming Ma

Department of Urology, The First Affiliated Hospital of Nanchang

University, Nanchang, 330006, Jiangxi

Province, People's Republic of China

EmailmmI5070835359@163.com
Background: SLFN11 has been found to regulate the development and progression of a variety of cancers and is associated with drug therapy, while its role in clear cell renal cell carcinoma (ccRCC) remains unclear; therefore, the aim of this study was to investigate SLFN11 expression in ccRCC patients and its correlations with clinicopathological and immunological features.

Methods: Gene profiles of ccRCC and the clinicopathological information of patients were downloaded from the TCGA database. Microarrays from the GEO database were used as a validation set for SLFN11 expression, which was experimentally verified in renal cancer cell lines by quantitative polymerase chain reaction (qPCR); protein expression and methylation levels were obtained from the HPA database and the UALCAN database. ROC curves, Kaplan-Meier survival analysis and Cox analysis were used to assess the diagnostic and predictive value of SLFN11 in ccRCC. Protein-protein interaction (PPI) networks for SLFN11 were obtained from the STRING website, and the TISIDB and TIMER 2.0 databases were used to study the relationship between SLFN11 and immune infiltration in the tumour microenvironment (TME).

Results: SLFN11 was significantly overexpressed in ccRCC tissues and renal cancer cell lines, which may be closely related to its hypermethylation status $(\mathrm{P}<0.001)$. SLFN11 was positively correlated with a highly aggressive disease state, with the ROC curve showing an AUC value of 0.910 for SLFN11 in diagnosing ccRCC, and Kaplan-Meier and Cox analyses also revealed that upregulation of SLFN11 predicted a poor prognosis for ccRCC patients ( $\mathrm{P}$ $<0.05)$. In addition, enrichment analysis showed that SLFN11 was closely associated with immune-related signalling pathways, and further exploration comprehensively demonstrated strong positive correlations with tumour immune lymphocytes, immune checkpoint genes, chemokines and chemokine receptors.

Conclusion: Overall, our data analysis shows that SLFN11 is a strong diagnostic and prognostic biomarker for ccRCC and is also associated with immune infiltration in the TME. Keywords: SLFN11, clear cell renal cell carcinoma, diagnostic, prognosis, immune infiltration

\section{Introduction}

Renal cell carcinoma is one of the most common malignancies of the genitourinary system, accounting for 431,288 new cases and 179,368 patient deaths worldwide in 2020. ${ }^{1}$ Clear cell renal cell carcinoma (ccRCC), on the other hand, is the predominant pathological type of kidney cancer, accounting for approximately $80 \%$ of cases. ${ }^{2}$ Due to the lack of specific clinical manifestations in the early stages of the disease, many 
patients are already ineligible for radical surgery by the time that they are diagnosed, and many patients experience local recurrence or distant metastases even after radical surgery. Although many patients have the option of receiving targeted therapy or immunotherapy, effective long-term treatment responses are rare, and more robust biomarkers are urgently needed to address tumour mutations. ${ }^{3}$

The Schlafen (SLFN) gene family has a wide range of biological functions and has been shown to be involved in cell proliferation, induction of the immune response and inhibition of viral replication. ${ }^{4}$ SLFN11, a major member of the Schlafen family, is a 901-amino acid residue polypeptide with two primary structural domains. ${ }^{5}$ In previous studies, high SLFN11 expression was found to be a good independent prognostic factor for ovarian cancer patients treated with cisplatin and in KRAS exon 2 wild-type colorectal cancer patients after oxaliplatin-based adjuvant therapy. ${ }^{6,7}$ In addition, SLFN11 plays an important role in the pharmacological treatment of prostate cancer and Ewing sarcoma. ${ }^{8,9}$ In brief, SLFN11 is a key predictive biomarker for a wide range of cancer therapies and a prognostic marker for many cancer types. ${ }^{10}$

However, the role of SLFN11 in ccRCC patients is not yet known. Therefore, the aim of this study was to investigate SLFN11 expression in ccRCC patients and its predictive value for patient prognosis and to explore its relationships with immune characteristics in the tumour microenvironment (TME) and thus identify a valid diagnostic and prognostic biomarker and potential therapeutic target for ccRCC.

\section{Methods}

\section{TCGA and GEO Datasets}

We used the TCGA database (https://portal.gdc.cancer. gov/) to obtain clinical information on ccRCC patients, ${ }^{11}$ including SLFN11 expression in 539 tumour tissues and 72 paraneoplastic tissues, baseline characteristics, disease stage and survival prognostic information. We also downloaded RNA-Seq expression data for SLFN11 in ccRCC from the GEO database (https://www.ncbi.nlm.nih.gov/ gds/); ${ }^{12}$ GSE5300 included 56 tumour tissues and 6 normal kidney tissues, GSE105261 included 35 tumour samples and 9 normal samples, and GSE40435 and GSE53757 consisted of 101 and 72 paired samples, respectively.

\section{UALCAN and HPA Datasets}

Results from a protein expression analysis and methylation analysis of SLFN11 were obtained from the UALCAN database (http://ualcan.path.uab.edu/). ${ }^{13}$ The HPA database (https://www.proteinatlas.org/) was used to compare the protein expression of SLFN11 between normal kidney tissue and ccRCC tissue by immunohistochemical staining. $^{14}$

\section{Protein-Protein Interaction (PPI) and Enrichment Analyses}

The STRING website (https://string-db.org/) was used to search for SLFN11-binding proteins and to construct a PPI network; ${ }^{15}$ an adjusted $\mathrm{P}<0.05$ and $\mid \log 2$ (Fold Change) $\mid>$ 1 were considered indicative of differentially expressed genes (DEGs). Venn diagrams were then used for intersection analysis of TPM4-binding genes and DEGs in TCGA datasets. ${ }^{16}$ The top 300 genes most positively associated with SLFN11 were selected and then analysed using the R package "ClusterProfiler" for Gene Ontology (GO) enrichment and Kyoto Encyclopedia of Genes and Genomes (KEGG) pathway analyses. ${ }^{17}$ In addition, we analysed the potential correlations of SLFN11 with all genes using $\mathrm{R}$ software and further performed gene set enrichment analysis (GSEA), with a false discovery rate $($ FDR $)<0.25$ and normal $\mathrm{P}$ value $(\mathrm{NOM} \mathrm{p}$-Val $)<0.05$ implying statistical significance. ${ }^{18}$

\section{TISIDB and TIMER 2.0 Databases}

In this study, TIMER 2.0 (http://timer.cistrome.org/) was first used to perform a pan-cancer analysis of SLFN11, and then we explored the relevance of SLFN11 to six types of key tumour-infiltrating lymphocytes (TILs) in ccRCC in this database (B cells, CD4+ T cells, CD8+ T cells, neutrophils, macrophages, and dendritic cells). ${ }^{19}$ In addition, the relationships between SLFN11 expression and immunomodulators, chemokines, and chemokine receptors were explored through the TISIDB website (http://cis.hku.hk/ TISIDB/). ${ }^{20}$

\section{Quantitative Polymerase Chain Reaction (qPCR) Analysis}

Total RNA was first extracted from renal cancer cell lines (ACHN, 786-O, OSRC-2, and Caki-1) and normal renal tubular epithelial cells (HK-2) using TRIzol reagent (Cwbio, China), and then the RNA was reverse transcribed using the First-Strand cDNA Synthesis Kit (Qiagen, USA). qPCR was performed using the SYBR Real-Time PCR kit (Qiagen, USA), and a relative quantitative data analysis was performed with the $2^{-\Delta \Delta \mathrm{Ct}}$ method. The primer sequences 


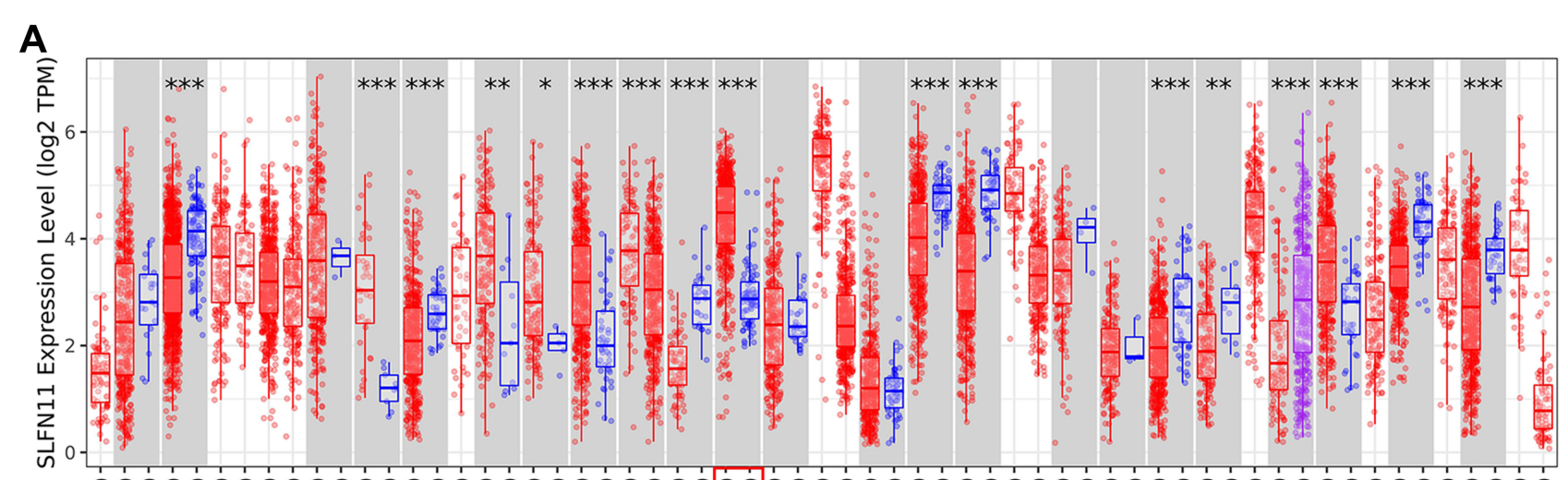

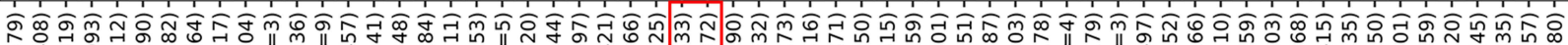
II II II

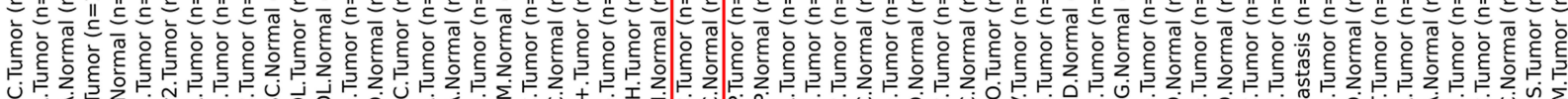

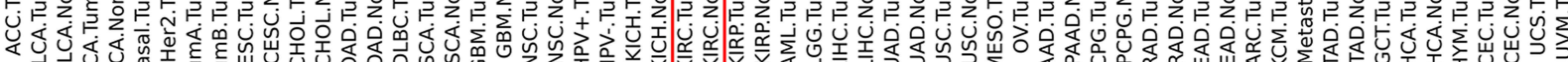

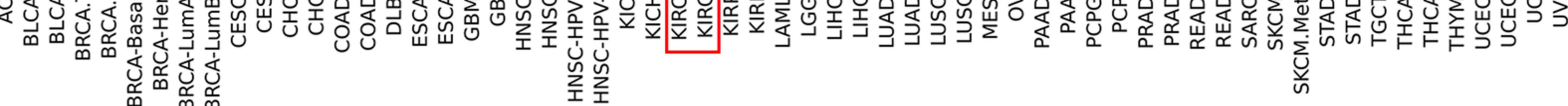

B

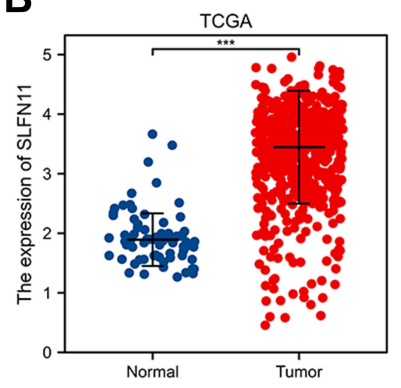

E

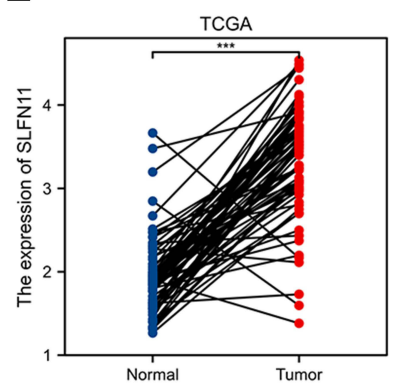

C

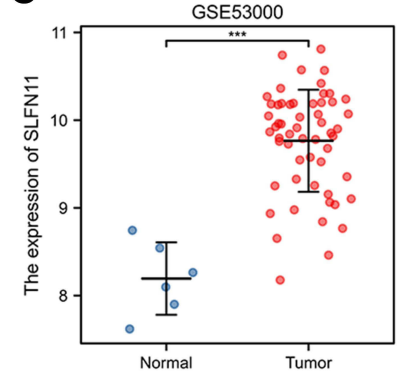

$\mathbf{F}$

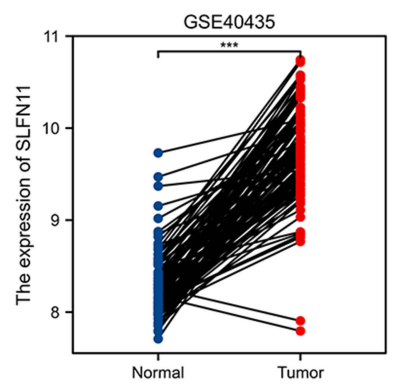

D

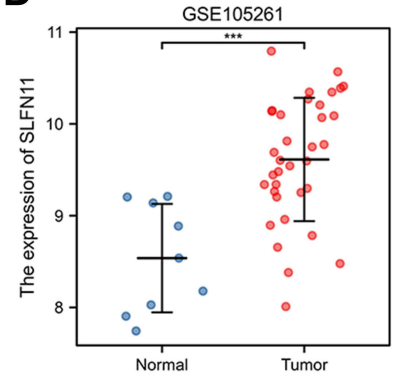

G

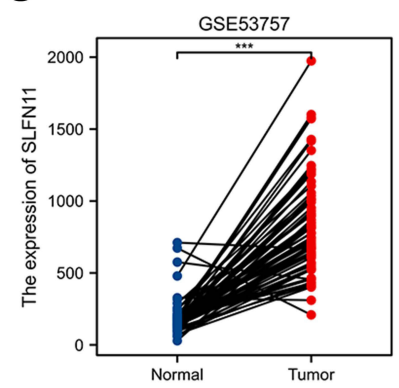

Figure I Expression levels of SLFNII in pan-cancer and ccRCC tissues from the TCGA and GEO databases. (A) Pan-cancer analysis of SLFNII. (B-G) Data from TCGA, GSE53000, GSEI0526I, GSE40435, and GSE53757 showed that SLFNII was highly expressed in ccRCC samples. ${ }^{*} \mathrm{p}<0.05$, **p $<0.01$, ***, p $<0.001$.

Abbreviations: SLFNII, Schlafen family member II; ccRCC, clear cell renal cell carcinoma.

for SLFN11 were 5'-TCGAAGGCTCAGGTGATT-3 (forward) and 5'-TGGGTAAGATGGTTCCACA-3' (reverse), and the reference gene was $\beta$-actin.

\section{Statistical Analyses}

All RNA-Seq gene expression data and clinical information obtained from the TCGA and GEO databases were analysed using $\mathrm{R}$ software (version 3.6.3). ${ }^{21}$ The Wilcoxon signed rank test and Wilcoxon rank sum test were used to compare differences between ccRCC tissue and normal kidney tissue, and ROC curves were examined using the pROC package. ${ }^{22}$ Kaplan-Meier analysis and Cox analysis were used to assess the effect of SLFN11 on survival, and Spearman correlation analysis was used to describe correlations between SLFN11 expression and TILs and other molecules. $\mathrm{P}<0.05$ was considered indicative of a statistically significant result. 
A

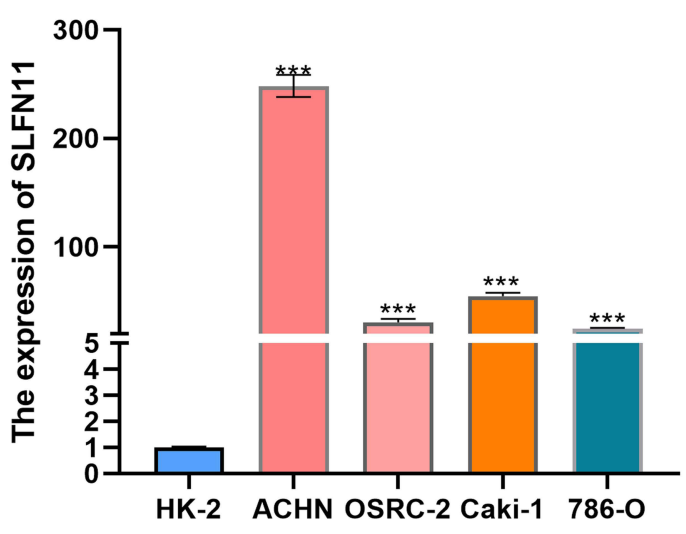

C

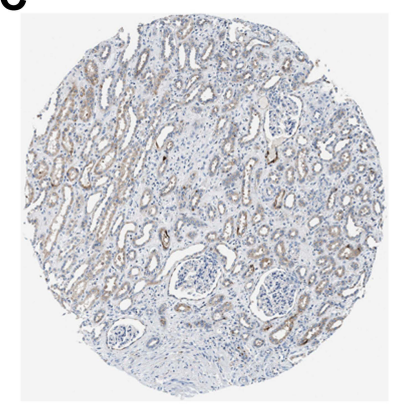

HPA023030

Normal

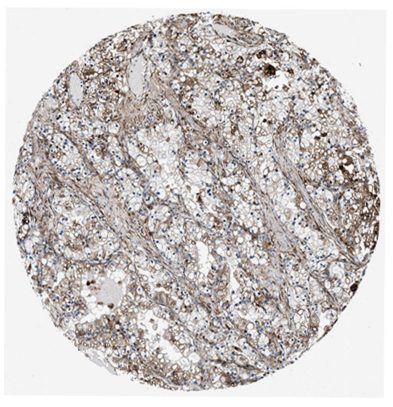

HPA023030

Tumor
B

Protein expression of SLFN11 in Clear cell RCC

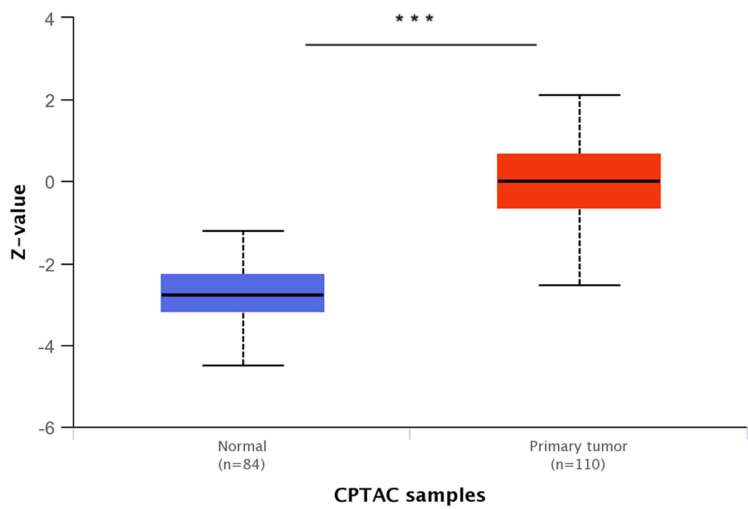

D

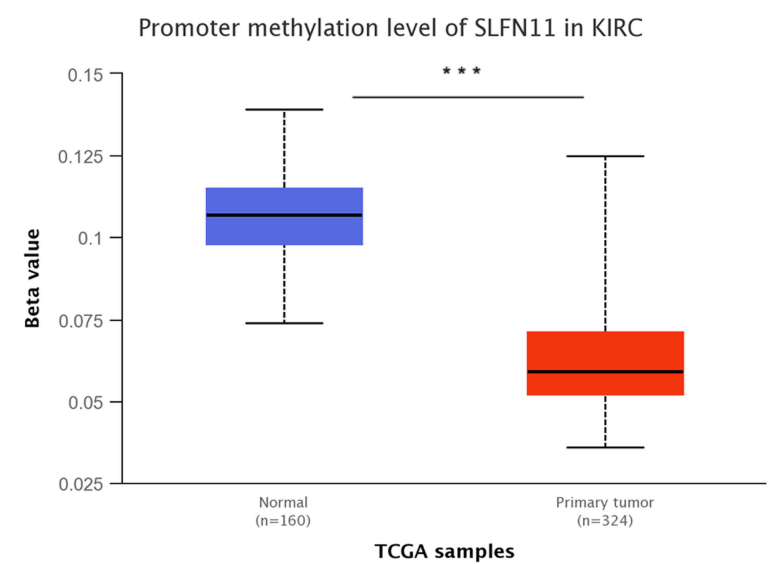

Figure 2 Expression levels of SLFNII in kidney cancer cell lines and ccRCC tissues and methylation levels in ccRCC tissues. (A) The mRNA expression of SLFNII was higher in renal cell carcinoma cell lines (ACHN, 786-O, OSRC-2, and Caki-I) than in normal renal tubular epithelial cells (HK-2). (B) SLFNII protein expression was higher in ccRCC tissues than in normal tissues. (C) Immunohistochemical staining of SLFNII in ccRCC tissues and normal kidney tissues. (D) SLFNI I is hypermethylated in ccRCC tissues relative to normal tissues. ${ }^{*} * \mathrm{p}<0.00 \mathrm{I}$.

Abbreviations: SLFNII, Schlafen family member II; ccRCC, clear cell renal cell carcinoma.

\section{Results}

\section{SLFNII is Highly Expressed in ccRCC Patients}

We first performed a pan-cancer analysis of SLFN11 in TIMER 2.0 and found that it was highly expressed in KIRC, CHOL, ESCA, GBM, HNSC and STAD, while its expression was low in $\mathrm{KICH}, \mathrm{BRCA}, \mathrm{COAD}$, LUAD, LUSC, PRAD, READ, THCA and UCEC (Figure 1A). Moreover, not only did the TCGA data show high SLFN11 expression in ccRCC tissues, but we also found similar results in the GEO database (Figures $1 \mathrm{~B}-\mathrm{G}$ ). On the other hand, the qPCR results revealed significantly higher levels of SLFN11 expression in renal cell carcinoma cell lines (ACHN, 786-O, OSRC-2, and Caki-1) than in normal renal tubular epithelial cells (HK-2) (Figure 2A). Similarly, at the protein level, SLFN11 expression was higher in ccRCC tissues than in paraneoplastic tissues (Figure 2B). Subsequently, we observed widespread expression of SLFN11 in ccRCC tissue by immunohistochemical staining, whereas in normal kidney tissue, SLFN11 was mainly expressed in tubular cells to some extent and was completely absent in glomerular cells (Figure 2C). To investigate the cause of high SLFN11 
Table I Clinical Characteristics of ccRCC Patients and the Relationship with SLFNII Expression

\begin{tabular}{|c|c|c|c|c|c|}
\hline Characteristic & Variable & $\mathbf{N}$ & Low Expression of SLFN I I & High Expression of SLFN I I & $P$ value \\
\hline \multirow[t]{2}{*}{ Age, n (\%) } & $\leq 60$ & $269(49.9 \%)$ & $146(27.1 \%)$ & $123(22.8 \%)$ & 0.053 \\
\hline & $>60$ & $270(50.1 \%)$ & $123(22.8 \%)$ & | 47 (27.3\%) & \\
\hline \multirow[t]{2}{*}{ Gender, n (\%) } & Female & $186(34.5 \%)$ & 97 (18\%) & 89 (16.5\%) & 0.506 \\
\hline & Male & $353(65.5 \%)$ & $172(3 \mid .9 \%)$ & I8I (33.6\%) & \\
\hline \multirow[t]{4}{*}{ T stage, n (\%) } & TI & $278(51.6 \%)$ & 153 (28.4\%) & 125 (23.2\%) & 0.002 \\
\hline & $\mathrm{T} 2$ & 71 (13.2\%) & $42(7.8 \%)$ & $29(5.4 \%)$ & \\
\hline & T3 & 179 (33.2\%) & 7I (13.2\%) & $108(20 \%)$ & \\
\hline & T4 & II (2\%) & $3(0.6 \%)$ & $8(1.5 \%)$ & \\
\hline \multirow[t]{2}{*}{ N stage, n (\%) } & No & $24 I(93.8 \%)$ & I2I (47.I\%) & 120 (46.7\%) & 0.468 \\
\hline & $\mathrm{NI}$ & $16(6.2 \%)$ & $6(2.3 \%)$ & $10(3.9 \%)$ & \\
\hline \multirow[t]{2}{*}{ M stage, $n(\%)$} & Mo & 428 (84.6\%) & 225 (44.5\%) & 203 (40.1\%) & $<0.001$ \\
\hline & MI & 78 (15.4\%) & $21(4.2 \%)$ & 57 (II.3\%) & \\
\hline \multirow[t]{4}{*}{ Histologic grade, n (\%) } & GI & $14(2.6 \%)$ & $12(2.3 \%)$ & $2(0.4 \%)$ & 0.005 \\
\hline & G2 & 235 (44.3\%) & 124 (23.4\%) & III (20.9\%) & \\
\hline & G3 & 207 (39\%) & 99 (18.6\%) & 108 (20.3\%) & \\
\hline & G4 & 75 (14.1\%) & $28(5.3 \%)$ & 47 (8.9\%) & \\
\hline \multirow[t]{4}{*}{ Pathologic stage, n (\%) } & Stage I & $272(50.7 \%)$ & I5I (28.2\%) & 121 (22.6\%) & $<0.001$ \\
\hline & Stage II & 59 (11\%) & 35 (6.5\%) & 24 (4.5\%) & \\
\hline & Stage III & $123(22.9 \%)$ & $56(10.4 \%)$ & $67(12.5 \%)$ & \\
\hline & Stage IV & $82(15.3 \%)$ & $25(4.7 \%)$ & $57(10.6 \%)$ & \\
\hline \multirow[t]{2}{*}{ OS event, n (\%) } & Alive & $366(67.9 \%)$ & $200(37.1 \%)$ & $166(30.8 \%)$ & 0.002 \\
\hline & Dead & 173 (32.1\%) & 69 (12.8\%) & 104 (19.3\%) & \\
\hline
\end{tabular}

Abbreviations: SLFNII, schlafen family member II; ccRCC, Clear cell renal cell carcinoma; OS, overall survival.

expression, we performed a methylation analysis and found that SLFN11 was significantly less methylated in ccRCC tissue than in normal kidney tissue (Figure 2D).

\section{The Relationships Between SLFNII and Clinicopathological Characteristics}

The clinical information obtained from TCGA for ccRCC patients and its relationship with SLFN11 expression is shown in Table 1. In addition, in Figures 3A-I, we can visually see that SLFN11 is significantly highly expressed in ccRCC patients with T3-T4 stage disease, a high pathological stage, distant metastases and fatal events. The ROC curve used to determine the diagnostic value of SLFN11 for ccRCC shows encouraging performance in terms of differentiating tumour from non-tumour tissues (Figure 4A). The efficacy of SLFN11 in diagnosing patients with ccRCC at different pathological stages is equally excellent, with AUC values of 0.893 and 0.936 in stages I-II and III-IV, respectively (Figures 4B and C).
On the other hand, in an assessment of the impact of SLFN11 on the survival of ccRCC patients, the results of the Kaplan-Meier analysis showed that patients with high SLFN11 expression had significantly shorter diseasespecific survival (DSS) and overall survival (OS) than those with low expression (Figure 4D and E). We further performed univariate and multivariate Cox regression analyses to verify the effect of SLFN11 on DSS and OS in ccRCC patients; the results suggested that pathological stage was an independent risk factor for DSS, and M-stage, histological grade and SLFN11 met the criteria as independent risk factors for both OS and DSS (Figures 4F-I).

\section{Analysis of the Potential Mechanism of SLFNII in ccRCC}

To explore the potential mechanisms of SLFN11 in ccRCC, we first performed a PPI network analysis using the STRING website. SLFN11 has been shown by 
A

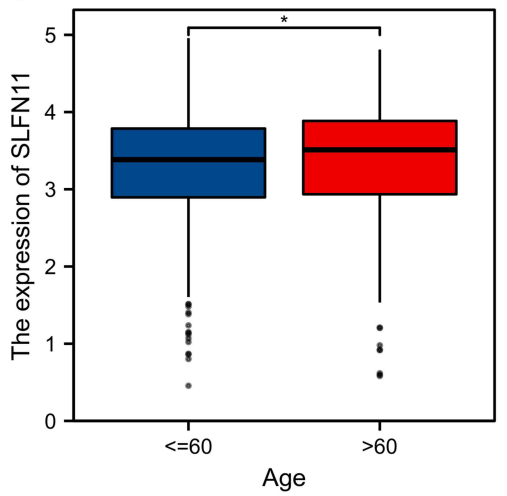

D

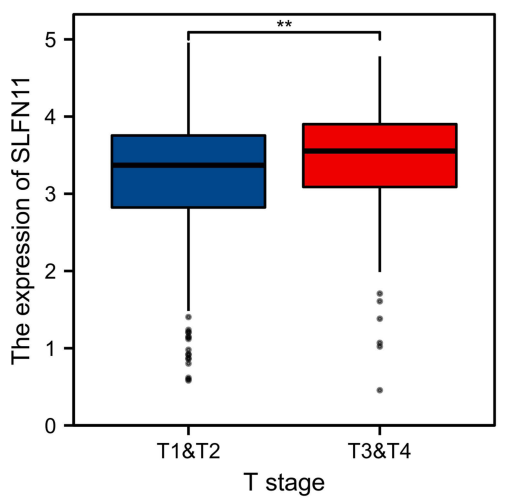

G

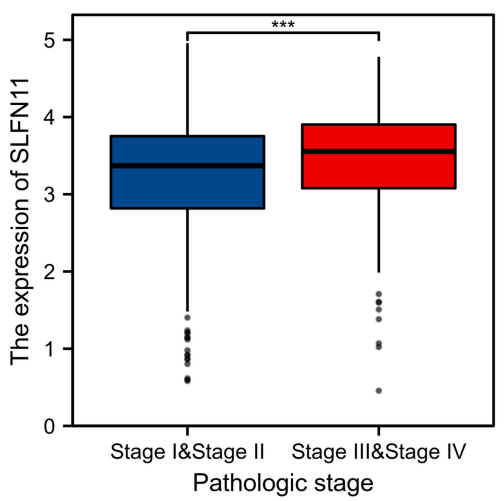

B

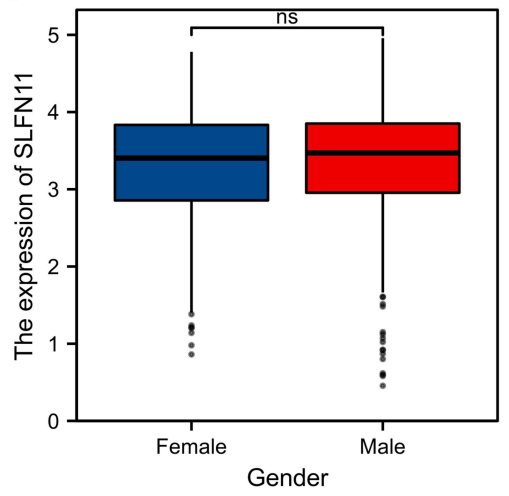

$\mathbf{E}$

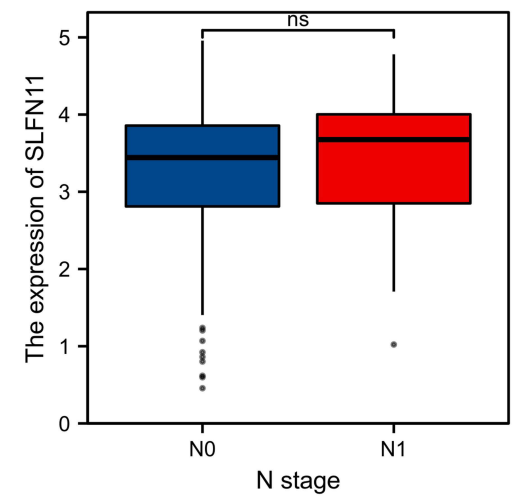

H

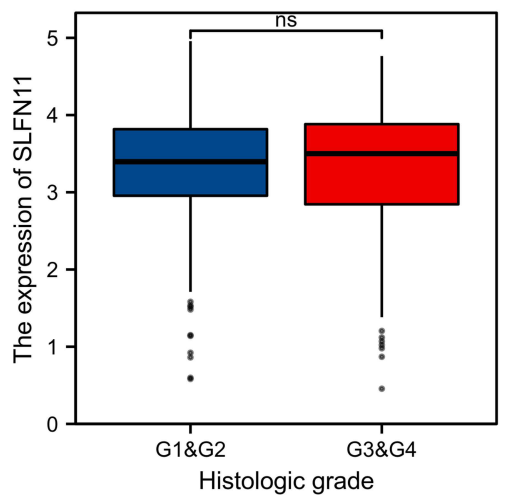

C

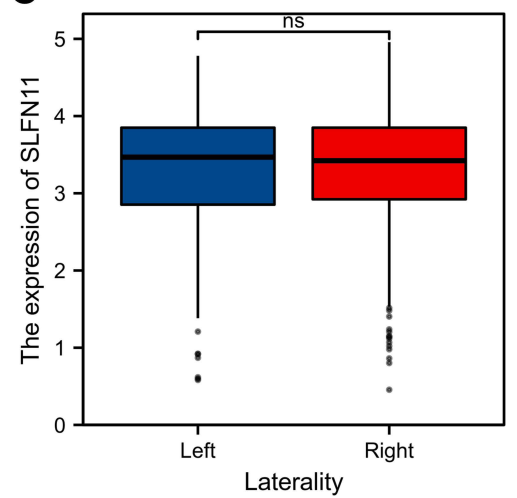

$\mathbf{F}$

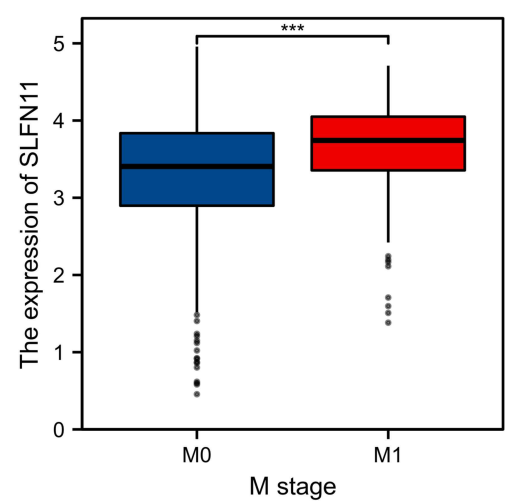

I

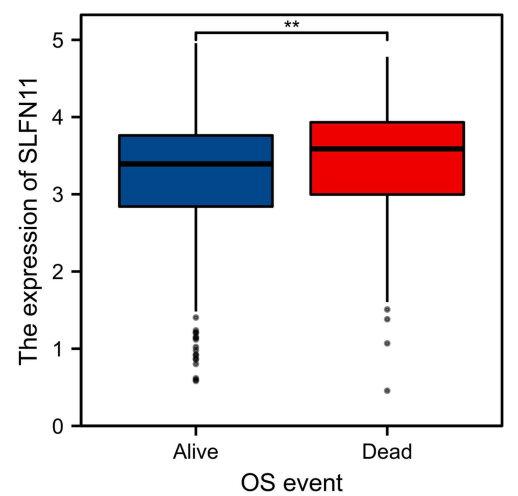

Figure 3 Relationships between SLFNII expression levels and clinicopathological features. High expression was observed in patients with an advanced age (A), T3-T4 stage disease (D), distant metastases (F), a high pathological stage $(\mathbf{G})$ and fatal events (I); no significant correlation with gender $(\mathbf{B})$, location $(\mathbf{C})$, lymph node metastasis $(\mathbf{E})$, or histological grading $(\mathbf{H})$ was identified. ${ }^{*} p<0.05$, ${ }^{* *} p<0.01$, ${ }^{* * *} p<0.001$.

Abbreviation: SLFNII, Schlafen family member II.

previous studies to bind directly to 19 protein molecules, which is also demonstrated in Figure 5A. We further performed an interaction analysis of DEGs in ccRCC with SLFN11-binding genes and showed that common members included SAMHD1, BST2, APOBEC3D, APOBEC3G, ETS1, and PVRIG (Figure 5B). In addition, these common genes were also found to correlate strongly with the expression levels of SLFN11 (Figure 5C).

To explore the potential functions and signalling pathways of SLFN11 in ccRCC, we analysed correlations between SLFN11 and other genes through the TCGA database, and the top 300 genes positively associated 


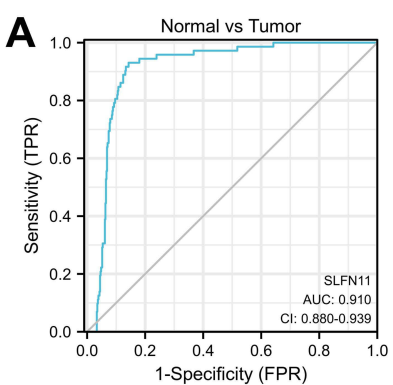

D

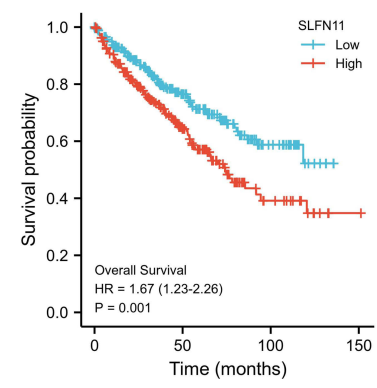

E

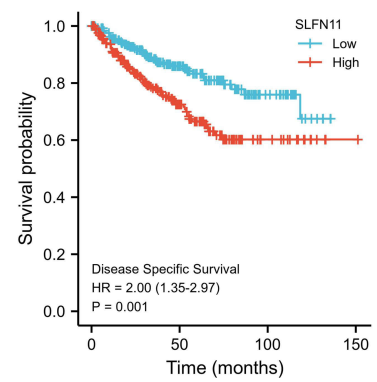

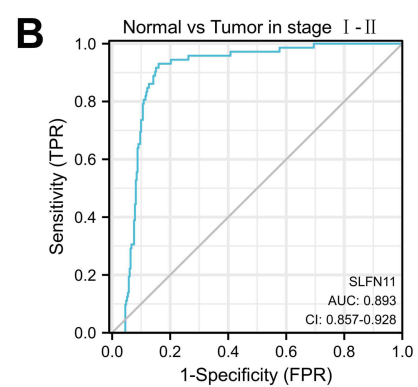

$\mathbf{F}$

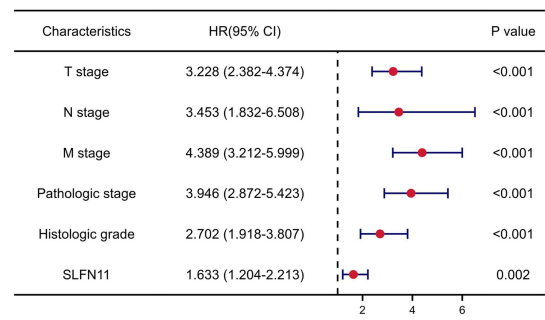

H

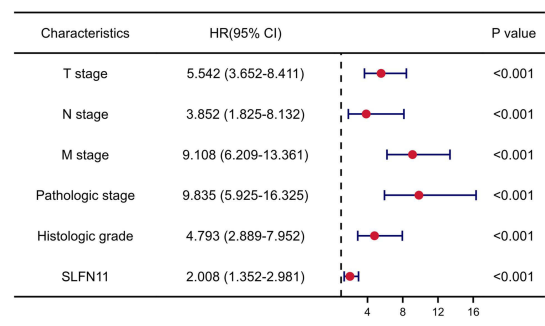

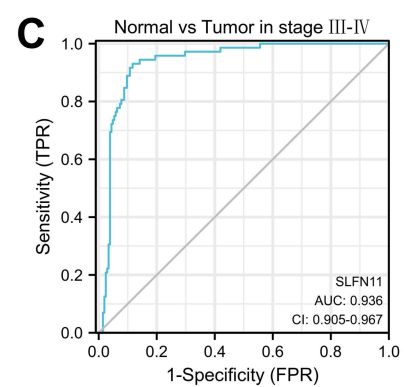

G

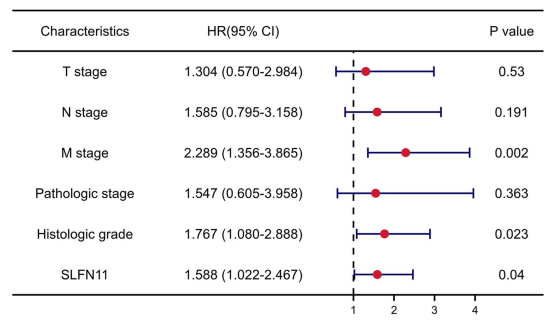

1

\begin{tabular}{|c|c|c|c|}
\hline Characteristics & $\mathrm{HR}(95 \% \mathrm{Cl})$ & & Pvalue \\
\hline T stage & $1.016(0.434-2.378)$ & $\phi$ & 0.972 \\
\hline N stage & $1.395(0.646-3.011)$ & po- & 0.396 \\
\hline M stage & $3.011(1.660-5.462)$ & ${ }_{1}$ & $<0.001$ \\
\hline Pathologic stage & $3.887(1.280-11.804)$ & & 0.017 \\
\hline Histologic grade & $1.975(1.011-3.858)$ & Ho- & 0.046 \\
\hline SLFN11 & $1.974(1.103-3.531)$ & $10-1$ & 0.022 \\
\hline
\end{tabular}

Figure 4 Diagnostic and predictive value of SLFNII. ROC curves of SLFNII expression in normal and tumour tissues (A) and subgroup analysis of patients with pathological stages I-II (B) and III-IV (C). Kaplan-Meier analysis showed that ccRCC patients with high SLFNII expression had shorter OS (D) and DSS (E) than those with low SLFNII expression. Forest plots of factors and their effects on OS by univariate (F) and multivariate (G) Cox regression analyses. Forest plots of factors and their effects on DSS by univariate $(\mathbf{H})$ and multivariate $(\mathbf{I})$ Cox regression analyses.

Abbreviations: SLFNII, Schlafen family member II; OS, overall survival; DSS, disease-specific survival.

with SLFN11 were extracted for subsequent enrichment analysis. GO analysis showed that SLFN11 may be associated with $\mathrm{T}$ cell activation and differentiation as well as lymphocyte activation and differentiation (Figure 6A). On the other hand, KEGG analysis showed that SLFN11 may be involved in the NOD-like receptor signalling pathway, chemokine signalling pathway, leukocyte transendothelial migration, $\mathrm{T}$ cell receptor signalling pathway, etc. (Figure 6B). The GSEA results showed that the KEGG pathways included chemokine signalling pathways, cytokine-cytokine receptor interactions, and primary immunodeficiency, and interleukin signalling, immunomodulatory interactions between lymphocytes and nonlymphoid cells, B-cell receptor signalling, and chemokine receptor binding to chemokines were enriched by the REACTOME signalling pathway (Figure 6C). Based on the results of these analyses, SLFN11 may have strong correlations with immune-related pathways in ccRCC.

\section{The Relationships Between SLFNII and Immune Characteristics}

To further understand the role that SLFN11 plays in immune infiltration in ccRCC, we first analysed the relationships between SLFN11 and several key TILs using the TIMER2.0 database, including B cells $(\mathrm{r}=0.247, \mathrm{P}=8.68 \mathrm{e}$ -08), CD4+ T cells $(r=0.402, P=2.79 \mathrm{e}-19), \mathrm{CD} 8+\mathrm{T}$ cells $(r=0.366, P=2.63 \mathrm{e}-15)$, macrophages $(r=0.43, P=1.24 \mathrm{e}$ $21)$, neutrophils $(\mathrm{r}=0.574, \mathrm{P}=1.43 \mathrm{e}-41)$, and dendritic 


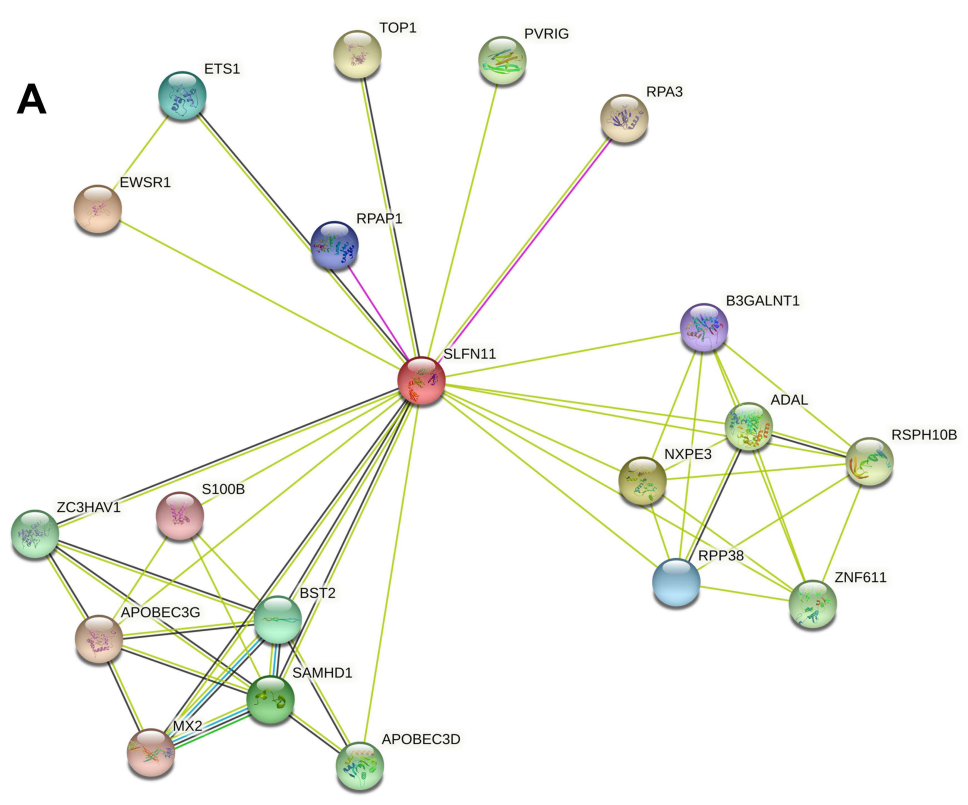

B
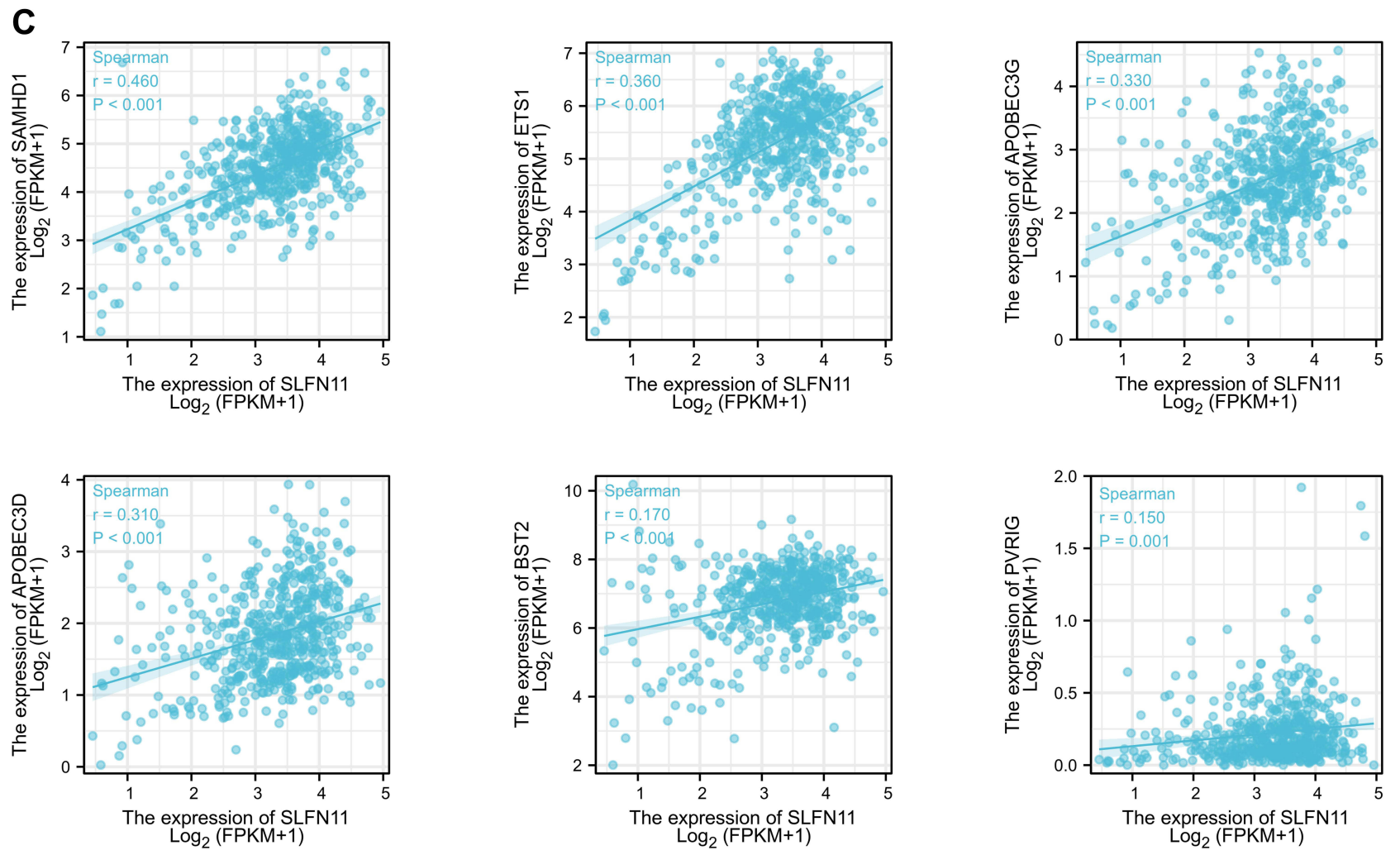

Figure 5 PPI network analysis of SLFNI I-related genes. (A) The PPI network of SLFNI I-binding proteins was obtained based on the STRING website. (B) An intersection analysis of SLFNII expression-correlated DEGs and TPM4-binding genes. (C) Correlation analysis between SLFNII expression and screened common genes, including SAMHDI, BST2, APOBEC3D, APOBEC3G, ETSI, and PVRIG.

Abbreviations: PPI, protein-protein interaction; SLFNII, Schlafen family member II; DEGs, differentially expressed genes.

cells $(\mathrm{r}=0.43, \mathrm{P}=6.32 \mathrm{e}-22)$ (Figure 7A). In addition, the associations between SLFN11 and multiple TILs in ccRCC are visualized in Figure 7B. Meanwhile, we explored correlations between SLFN11 and immunomodulatory genes in ccRCC, and the results showed positive correlations between SLFN11 and CD86, CTLA4, CD244, CD48, 

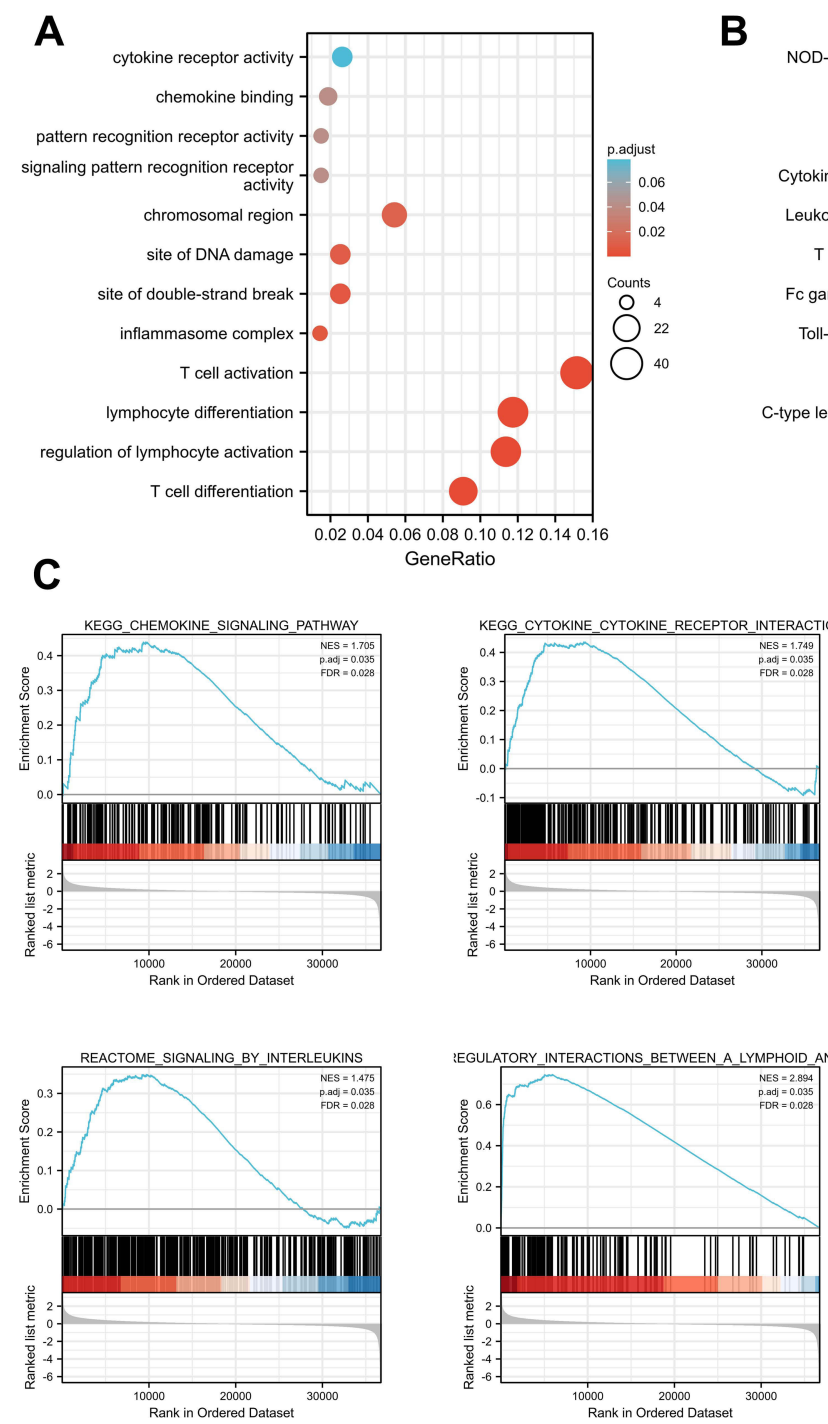

B

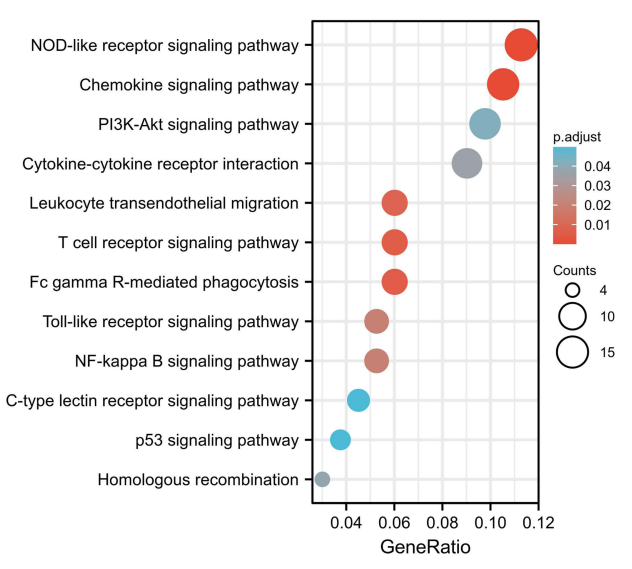

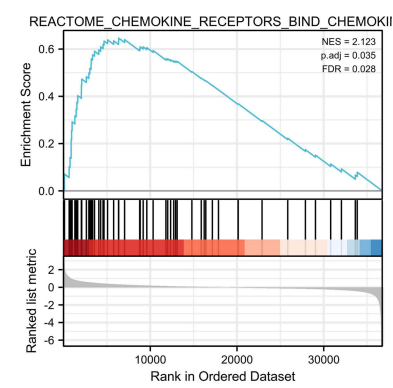
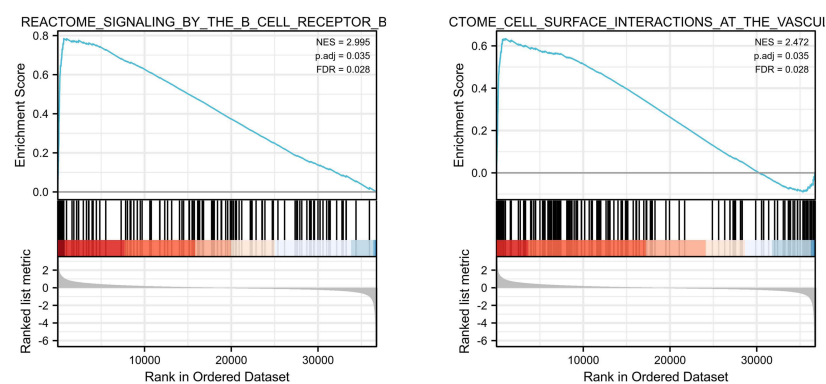

Figure 6 Enrichment analysis of SLFNII expression-correlated DEGs in ccRCC. (A) GO enrichment analysis. (B) KEGG enrichment analysis. (C) Enrichment plots by GSEA. KEGG pathways included chemokine signalling pathways, cytokine-cytokine receptor interactions, and primary immunodeficiency; the REACTOME signalling pathway included chemokine receptor binding to chemokines, interleukin signalling, immunomodulatory interactions between lymphocytes and nonlymphoid cells, B-cell receptor signalling and interaction of the cell surface with the vessel wall.

Abbreviations: SLFNI I, Schlafen family member II; DEGs, differentially expressed genes; ccRCC, clear cell renal cell carcinoma; GO, Gene Ontology; KEGG, Kyoto Encyclopedia of Genes and Genomes; GSEA, gene set enrichment analysis.

CD27, CD40 and so on (Figure 7C). Interestingly, these genes have been shown in previous studies to be relevant genes indicating ccRCC characteristics and may play an important role in the immunomodulation of ccRCC. $^{23}$ Finally, the potential associations of SLFN11 with chemokines and chemokine receptors in ccRCC were investigated on the TISIDB website, and the results demonstrated closer associations of SLFN11 with CXCL5 CXCL11, CXCL10, CXCL13, CXCR3, CXCR4, CXCR5, CXCR6, CCR5, and CCR6 (shown in Figures 8A-C). As these molecules are upregulated in ccRCC and are poor prognostic factors for patients, they may be involved in the role of SLFN11 in ccRCC. $^{24-27}$

\section{Discussion}

In the present study, we analysed SLFN11 expression and the clinical information of ccRCC patients obtained from public databases, and the results showed that SLFN11 expression was significantly higher in ccRCC tissues than in normal paracancerous tissues at both the mRNA and 


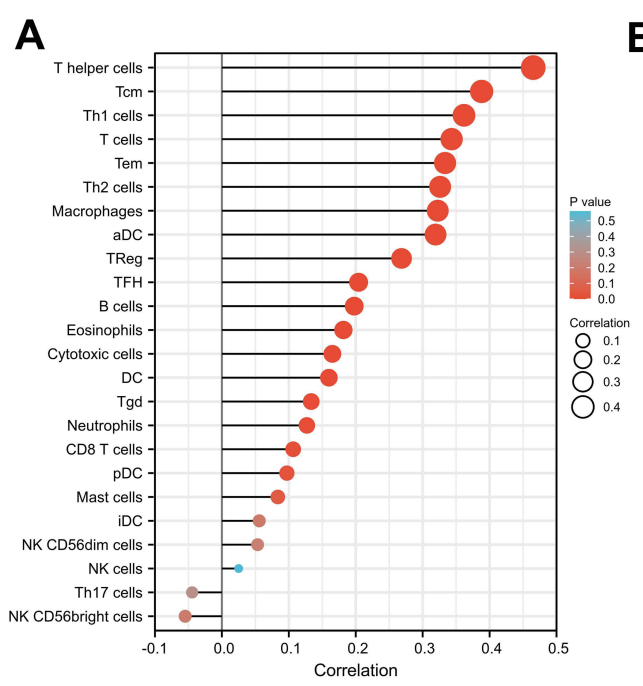

B
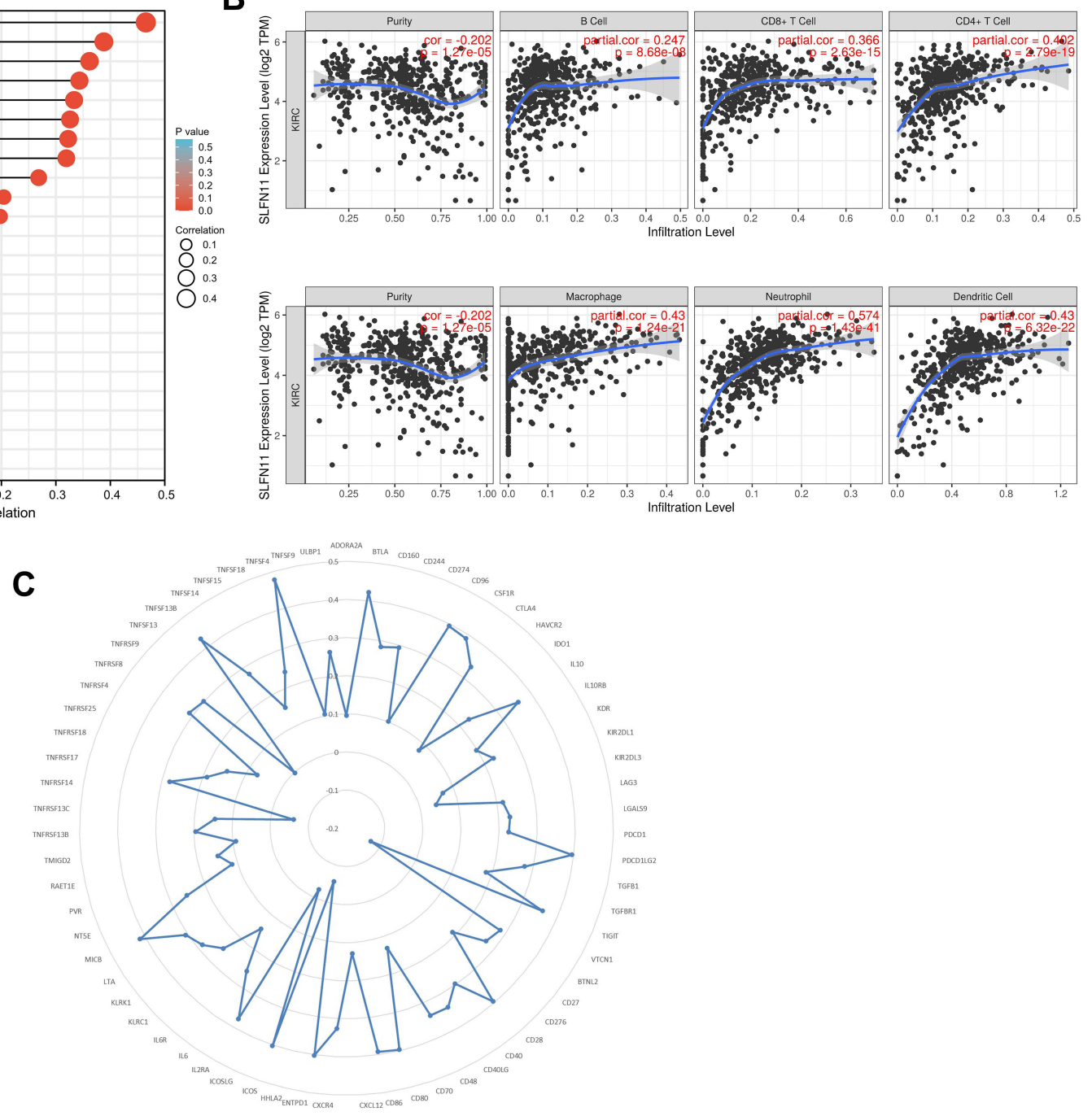

Figure 7 Correlation analysis of SLFNII expression with TILs and immunomodulatory genes in ccRCC. (A) TIL scores were associated with SLFNII expression. (B) Correlation analysis of SLFNII expression with immune infiltration levels of B cells, CD8+ T cells, CD4+ T cells, macrophages, neutrophils, and myeloid dendritic cells. (C) Correlations between SLFNII expression and immunomodulatory genes.

Abbreviations: SLFNII, Schlafen family member II; TILs, tumour immune cells; ccRCC, clear cell renal cell carcinoma.

protein levels. The ROC curve visually demonstrates the diagnostic value of SLFN11 for ccRCC, and the KaplanMeier survival analysis and Cox regression analysis suggested that upregulated SLFN11 predicts poor DSS and OS in ccRCC patients. Furthermore, we explored the relationship between SLFN11 and immune infiltration in ccRCC.

SLFN11, a putative DNA-RNA helicase, has been shown to increase tumour cells' sensitivity to DNA-damaging agents and poly(ADP-ribose) polymerase inhibitors, ${ }^{28,29}$ and downregulation of SLFN11 is strongly associated with drug resistance in multiple tumours. ${ }^{30,31}$ One study found that SLFN11 overexpression resulted in longer radiological progression-free survival when platinum-based chemotherapeutic agents were used in patients with castration-resistant prostate cancer. ${ }^{8}$ Similarly, Zhou et al found that upregulation of SLFN11 inhibited the proliferation, migration and invasion of hepatocellular carcinoma (HCC) cells, and unfortunately, SLFN11 presented low expression in $\mathrm{HCC}$, thus correlating with a shorter OS and higher recurrence rate in HCC patients. ${ }^{32}$ Kagami et al also found that higher levels of SLFN11 were independently associated with a good prognosis for oesophageal squamous cell carcinoma patients. ${ }^{33}$ In addition, high SLFN11 expression was also found to inhibit the growth of gastric and colorectal cancer cells, and SLFN11 increases resistance to cisplatin in these patients due to overmethylation..$^{34,35}$ However, in contrast to previous studies, 

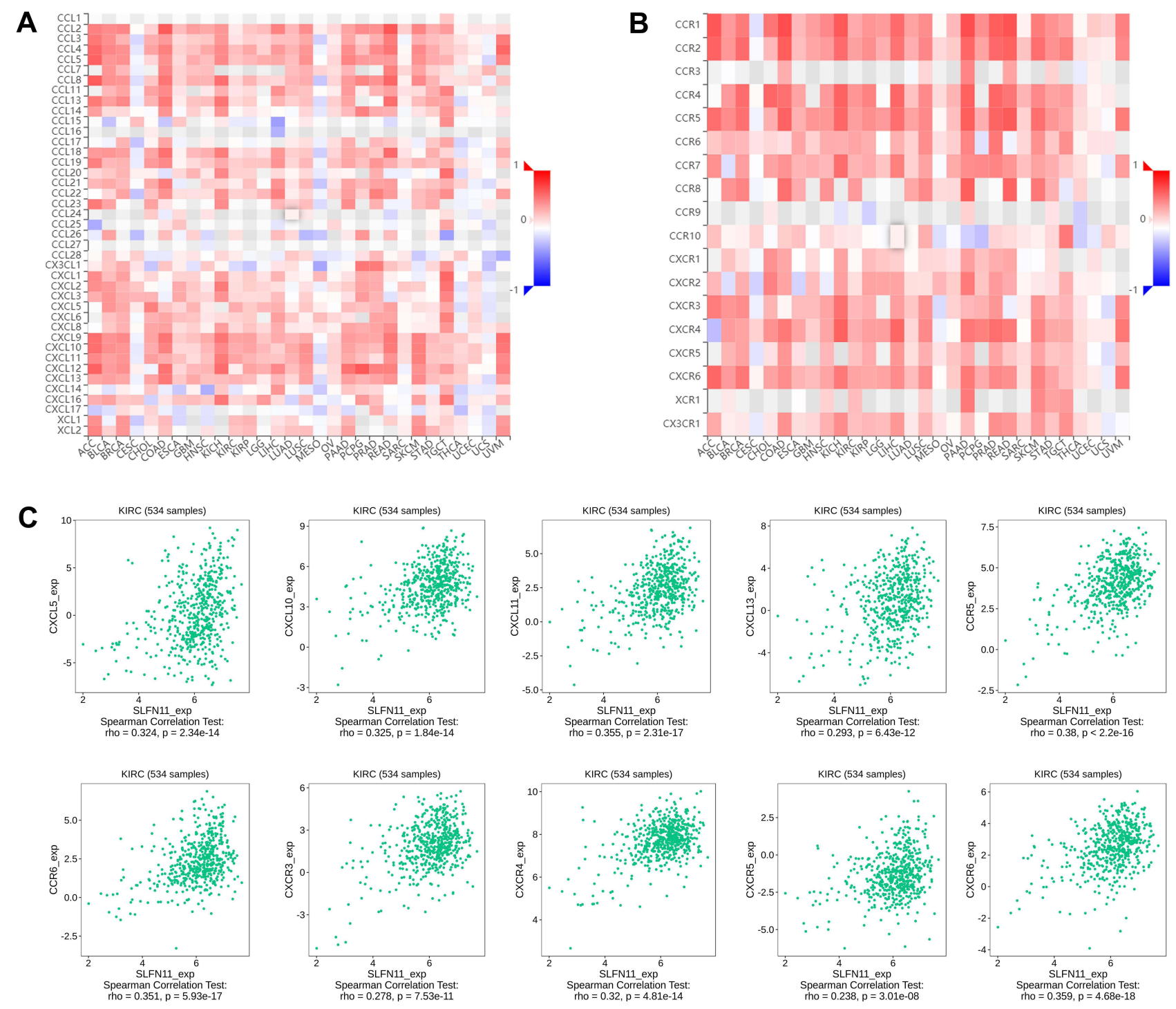

Figure 8 Correlation analysis of SLFNII expression with chemokines and chemokine receptors based on the TISIDB database. Plot of SLFNII expression versus chemokines (A) and chemokine receptors (B) in multiple cancer types. (C) SLFNII expression was positively correlated with the infiltration levels of CXCL5, CXCLI0, CXCLII, CXCLI3, CCR5, CCR6, CXCR3, CXCR4, CXCR5 and CXCR5.

Abbreviation: SLFNII, Schlafen family member II.

we found that SLFN11 was significantly upregulated in ccRCC due to hypomethylation and was also a detrimental factor affecting patient prognosis. At the same time, although SLFN11 upregulation in patients with lymph node metastases and high histological grades was not significant due to the unbalanced nature of the sample, high SLFN11 levels were clearly associated with more aggressive clinicopathological characteristics. Furthermore, SLFN11 was also found to have good diagnostic value for ccRCC by ROC curves. These results suggest that SLFN11 may have potential as a powerful diagnostic and prognostic biomarker for patients with ccRCC.
To understand the potential mechanism of action of SLFN11, we analysed the relationships between SLFN11 and other DEGs in ccRCC; the results suggest that SLFN11 has the greatest potential to interact with genes such as SAMHD1 and ETS1. Notably, SAMHD1 has been found to play an important role in the cell cycle, cancer, and innate immunity. ${ }^{36,37}$ Subsequently, we performed biological process analysis and signalling pathway enrichment analysis of SLFN11 and found that it is associated with the activation and differentiation of immune cells and may exert biological functions through immune-related pathways. Recent studies have revealed that SLFN11 also showed high expression in 
breast cancer patients with characteristics of triple-negative breast cancer, was closely associated with immune activation, and predicted a poorer prognosis in the short term. ${ }^{38}$ Based on these results, SLFN11 can reasonably be hypothesized to be involved in immune regulation in the TME of ccRCC patients.

Cancer development and progression are often accompanied by changes in the surrounding stroma, and immune cells, an important component of the tumour stroma, have been repeatedly shown to contribute to tumour progression when present in the TME. ${ }^{39}$ In the present study, we found that SLFN11 was significantly associated with tumourinfiltrating immune cells such as T cells, macrophages, neutrophils, and dendritic cells in ccRCC, which seems to further validate the role of SLFN11 in immune cell activation and differentiation. In addition, we found strong correlations between SLFN11 and some immune checkpoint genes. Among these molecules, high CTLA4 expression was shown to be associated with poorer OS and DSS in ccRCC patients, ${ }^{40}$ and CD244 was also found to contribute to the overall immune-suppressive environment. ${ }^{41}$ The expression of chemokines and their receptors in malignant tumours often determines leukocyte recruitment and activation, angiogenesis, cancer cell proliferation and disease metastasis, ${ }^{42}$ In ccRCC, CXCL13 promotes tumour cell proliferation and migration by binding to CXCR5 and activating the PI3K/ AKT/mTOR signalling pathway, and higher levels of CXCR3/4/5/6 expression are also associated with poor OS in patients. ${ }^{24,43}$ However, our study found that SLFN11 was positively correlated with these molecules to some extent. Thus, from the above results, SLFN11 likely plays some critical role in the TME of ccRCC.

To our knowledge, this is the first exploration of SLFN11 in ccRCC patients. However, we only investigated its potential signalling pathway and relationships with immune features in the TME, and the exact mechanism of its action is unclear; therefore, subsequent studies should be performed to address this issue.

\section{Conclusion}

Our data analysis showed that SLFN11 was significantly highly expressed in ccRCC, which may be due to hypomethylation. Furthermore, SLFN11 is a strong diagnostic and prognostic biomarker for ccRCC and is also closely associated with immune infiltration in the TME, thus representing a potential therapeutic target in ccRCC in the future.

\section{Data Sharing Statement}

RNA-seq and clinical information for ccRCC patients are available from both the TCGA database (https://portal.gdc. cancer.gov/) and the GEO database (https://www.ncbi.nlm. nih.gov/gds/).

\section{Ethics Statement}

The Ethics Committee of the First Affiliated Hospital of Nanchang University concluded that no ethical review was required for this study due to the fact that the patient data for this study were obtained from public databases and that these data had received informed consent from patients and ethical approval from the respective institutional ethics committees prior to disclosure.

\section{Funding}

This study is supported by the key project of science and technology research of Jiangxi Provincial education department. Fund No. GJJ190030. .

\section{Disclosure}

All authors declare that they have no conflicts of interest related to this work or this manuscript.

\section{References}

1. Sung H, Ferlay J, Siegel RL, et al. Global cancer statistics 2020: GLOBOCAN estimates of incidence and mortality worldwide for 36 cancers in 185 countries. CA Cancer J Clin. 2021;71(3):209-249.

2. Qin S, Shi X, Wang C, Jin P, Ma F. Transcription factor and miRNA interplays can manifest the survival of ccRCC patients. Cancers. 2019;11(11):1668. doi:10.3390/cancers11111668

3. Deleuze A, Saout J, Dugay F, et al. Immunotherapy in renal cell carcinoma: the future is now. Int $J$ Mol Sci. 2020;21(7):2532. doi:10.3390/ijms 21072532

4. Mavrommatis E, Fish EN, Platanias LC. The schlafen family of proteins and their regulation by interferons. J Interferon Cytokine Res. 2013;33(4):206-210. doi:10.1089/jir.2012.0133

5. Murai J, Thomas A, Miettinen M, Pommier Y. Schlafen 11 (SLFN11), a restriction factor for replicative stress induced by DNA-targeting anti-cancer therapies. Pharmacol Ther. 2019;201:94-102. doi:10.1016/j. pharmthera.2019.05.009

6. Zoppoli G, Regairaz M, Leo E, et al. Putative DNA/RNA helicase Schlafen-11 (SLFN11) sensitizes cancer cells to DNA-damaging agents. Proc Natl Acad Sci U S A. 2012;109(37):15030-15035. doi:10.1073/pnas.1205943109

7. Deng Y, Cai Y, Huang Y, et al. High SLFN11 expression predicts better survival for patients with KRAS exon 2 wild type colorectal cancer after treated with adjuvant oxaliplatin-based treatment. $B M C$ Cancer. 2015;15(1):833. doi:10.1186/s12885-015-1840-6

8. Conteduca V, Ku SY, Puca L, et al. SLFN11 expression in advanced prostate cancer and response to platinum-based chemotherapy. Mol Cancer Ther. 2020;19(5):1157-1164. doi:10.1158/1535-7163.MCT19-0926 
9. Tang SW, Bilke S, Cao L, et al. SLFN11 is a transcriptional target of EWS-FLI1 and a determinant of drug response in Ewing sarcoma. Clin Cancer Res. 2015;21(18):4184-4193. doi:10.1158/1078-0432. CCR-14-2112

10. Zhang B, Ramkumar K, Cardnell RJ, et al. A wake-up call for cancer DNA damage: the role of Schlafen 11 (SLFN11) across multiple cancers. Br J Cancer. 2021:1-8. doi:10.1038/s41416-021-01476-w

11. Tomczak K, Czerwińska P, Wiznerowicz M. The Cancer Genome Atlas (TCGA): an immeasurable source of knowledge. Contemp Oncol. 2015;19(1a):A68-A77.

12. Clough E, Barrett T. The gene expression omnibus database. Methods Mol Biol. 2016;1418:93-110.

13. Chandrashekar DS, Bashel B, Balasubramanya SAH, et al. UALCAN: a portal for facilitating tumor subgroup gene expression and survival analyses. Neoplasia. 2017;19(8):649-658. doi:10.1016/j. neo.2017.05.002

14. Uhlen M, Zhang C, Lee S, et al. A pathology atlas of the human cancer transcriptome. Science. 2017;357(6352):6352. doi:10.1126/ science.aan 2507

15. Szklarczyk D, Franceschini A, Kuhn M, et al. The STRING database in 2011: functional interaction networks of proteins, globally integrated and scored. Nucleic Acids Res. 2011;39(Database):D561D568. doi:10.1093/nar/gkq973

16. Bardou P, Mariette J, Escudié F, Djemiel C, Klopp C. Jvenn: an interactive venn diagram viewer. BMC Bioinform. 2014;15(1):293. doi:10.1186/1471-2105-15-293

17. Yu G, Wang LG, Han Y, He QY. ClusterProfiler: an R package for comparing biological themes among gene clusters. OMICS. 2012;16 (5):284-287. doi:10.1089/omi.2011.0118

18. Subramanian A, Tamayo P, Mootha VK, et al. Gene set enrichment analysis: a knowledge-based approach for interpreting genome-wide expression profiles. Proc Natl Acad Sci U S A. 2005;102 (43):15545-15550. doi:10.1073/pnas.0506580102

19. Li T, Fu J, Zeng Z, et al. TIMER2.0 for analysis of tumor-infiltrating immune cells. Nucleic Acids Res. 2020;48(W1):W509-W514. doi:10.1093/nar/gkaa407

20. $\mathrm{Ru} \mathrm{B}$, Wong $\mathrm{CN}$, Tong $\mathrm{Y}$, et al. TISIDB: an integrated repository portal for tumor-immune system interactions. Bioinformatics. 2019;35(20):4200-4202. doi:10.1093/bioinformatics/btz210

21. Kruppa J, Jung K. Automated multigroup outlier identification in molecular high-throughput data using bagplots and gemplots. $B M C$ Bioinform. 2017;18(1):232. doi:10.1186/s12859-017-1645-5

22. Robin X, Turck N, Hainard A, et al. pROC: an open-source package for $\mathrm{R}$ and $\mathrm{S}+$ to analyze and compare ROC curves. BMC Bioinform. 2011;12(1):77. doi:10.1186/1471-2105-12-77

23. Tronik-Le Roux D, Sautreuil M, Bentriou M, et al. Comprehensive landscape of immune-checkpoints uncovered in clear cell renal cell carcinoma reveals new and emerging therapeutic targets. Cancer Immunol Immunother. 2020;69(7):1237-1252. doi:10.1007/s00262020-02530-x

24. Wu Z, Zhang Y, Chen X, et al. Characterization of the prognostic values of the CXCR1-7 in Clear Cell Renal Cell Carcinoma (ccRCC) microenvironment. Front Mol Biosci. 2020;7:601206. doi:10.3389/ fmolb.2020.601206

25. Esteban E, Exposito F, Crespo G, et al. Circulating levels of the interferon- $\gamma$-regulated chemokines CXCL10/CXCL11, IL-6 and HGF predict outcome in metastatic renal cell carcinoma patients treated with antiangiogenic therapy. Cancers. 2021;13(11):2849. doi: $10.3390 /$ cancers 13112849

26. Giuliano S, Dufies M, Ndiaye PD, et al. Resistance to lysosomotropic drugs used to treat kidney and breast cancers involves autophagy and inflammation and converges in inducing CXCL5. Theranostics. 2019;9(4):1181-1199. doi:10.7150/thno.29093
27. Dai S, Zeng H, Liu Z, et al. Intratumoral CXCL13 + CD8 + T cell infiltration determines poor clinical outcomes and immunoevasive contexture in patients with clear cell renal cell carcinoma. $J$ Immunother Cancer. 2021;9(2):e001823. doi:10.1136/jitc-2020001823

28. Luan J, Gao X, Hu F, Zhang Y, Gou X. SLFN11 is a general target for enhancing the sensitivity of cancer to chemotherapy (DNA-damaging agents). $J$ Drug Target. 2020;28(1):33-40. doi:10.1080/1061186X.2019.1616746

29. Lok BH, Gardner EE, Schneeberger VE, et al. PARP inhibitor activity correlates with SLFN11 expression and demonstrates synergy with temozolomide in small cell lung cancer. Clin Cancer Res. 2017;23(2):523-535. doi:10.1158/1078-0432.CCR-16-1040

30. Mao S, Chaerkady R, Yu W, et al. Resistance to pyrrolobenzodiazepine dimers is associated with SLFN11 downregulation and can be reversed through inhibition of ATR. Mol Cancer Ther. 2021;20 (3):541-552. doi:10.1158/1535-7163.MCT-20-0351

31. Berns K, Berns A. Awakening of "Schlafen11" to tackle chemotherapy resistance in SCLC. Cancer Cell. 2017;31(2):169-171. doi:10.1016/j.ccell.2017.01.013

32. Zhou C, Liu C, Liu W, et al. SLFN11 inhibits hepatocellular carcinoma tumorigenesis and metastasis by targeting RPS4X via mTOR pathway. Theranostics. 2020;10(10):4627-4643. doi:10.7150/ thno.42869

33. Kagami T, Yamade M, Suzuki T, et al. The first evidence for SLFN11 expression as an independent prognostic factor for patients with esophageal cancer after chemoradiotherapy. BMC Cancer. 2020;20 (1):1123. doi:10.1186/s12885-020-07574-x

34. He T, Zhang M, Zheng R, et al. Methylation of SLFN11 is a marker of poor prognosis and cisplatin resistance in colorectal cancer. Epigenomics. 2017;9(6):849-862. doi:10.2217/epi-2017-0019

35. Peng Y, Wang L, Wu L, Zhang L, Nie G, Guo M. Methylation of SLFN11 promotes gastric cancer growth and increases gastric cancer cell resistance to cisplatin. $J$ Cancer. 2019;10(24):6124-6134. doi:10.7150/jca.32511

36. Mauney $\mathrm{CH}$, Hollis T. SAMHD1: recurring roles in cell cycle, viral restriction, cancer, and innate immunity. Autoimmunity. 2018;51 (3):96-110. doi:10.1080/08916934.2018.1454912

37. Zhang Z, Zheng L, Yu Y, et al. Involvement of SAMHD1 in dNTP homeostasis and the maintenance of genomic integrity and oncotherapy (Review). Int J Oncol. 2020;56(4):879-888.

38. Isnaldi E, Ferraioli D, Ferrando L, et al. Schlafen-11 expression is associated with immune signatures and basal-like phenotype in breast cancer. Breast Cancer Res Treat. 2019;177(2):335-343. doi:10.1007/ s10549-019-05313-w

39. Hinshaw DC, Shevde LA. The tumor microenvironment innately modulates cancer progression. Cancer Res. 2019;79(18):4557-4566. doi:10.1158/0008-5472.CAN-18-3962

40. Liao G, Wang P, Wang Y. Identification of the prognosis value and potential mechanism of immune checkpoints in renal clear cell carcinoma microenvironment. Front Oncol. 2021;11:720125. doi:10.3389/ fonc. 2021.720125

41. Agresta L, Lehn M, Lampe K, et al. CD244 represents a new therapeutic target in head and neck squamous cell carcinoma. $J$ Immunother Cancer. 2020;8(1):e000245. doi:10.1136/jitc-2019000245

42. Mollica Poeta V, Massara M, Capucetti A, Bonecchi R. Chemokines and chemokine receptors: new targets for cancer immunotherapy. Front Immunol. 2019;10:379. doi:10.3389/fimmu.2019.00379

43. Zheng Z, Cai Y, Chen H, et al. CXCL13/CXCR5 axis predicts poor prognosis and promotes progression through $\mathrm{PI} 3 \mathrm{~K} / \mathrm{AKT} / \mathrm{mTOR}$ pathway in clear cell renal cell carcinoma. Front Oncol. 2019;8:682. doi:10.3389/fonc. 2018.00682 


\section{Publish your work in this journal}

The International Journal of General Medicine is an international, peer-reviewed open-access journal that focuses on general and internal medicine, pathogenesis, epidemiology, diagnosis, monitoring and treatment protocols. The journal is characterized by the rapid reporting of reviews, original research and clinical studies

across all disease areas. The manuscript management system is completely online and includes a very quick and fair peer-review system, which is all easy to use. Visit http://www.dovepress.com/ testimonials.php to read real quotes from published authors.

Submit your manuscript here: https://www.dovepress.com/international-journal-of-general-medicine-journal 